This item was submitted to Loughborough's Research Repository by the author.

Items in Figshare are protected by copyright, with all rights reserved, unless otherwise indicated.

\title{
Finite-time relative pose tracking control for uncertain spacecraft rendezvous and docking
}

PLEASE CITE THE PUBLISHED VERSION

https://doi.org/10.1109/CCDC52312.2021.9602803

PUBLISHER

IEEE

VERSION

AM (Accepted Manuscript)

\section{PUBLISHER STATEMENT}

(c) 2021 IEEE. Personal use of this material is permitted. Permission from IEEE must be obtained for all other uses, in any current or future media, including reprinting/republishing this material for advertising or promotional purposes, creating new collective works, for resale or redistribution to servers or lists, or reuse of any copyrighted component of this work in other works.

\section{LICENCE}

All Rights Reserved

\section{REPOSITORY RECORD}

Sun, Liang, Jinwei Wang, and Jingjing Jiang. 2021. "Finite-time Relative Pose Tracking Control for Uncertain Spacecraft Rendezvous and Docking". Loughborough University. https://hdl.handle.net/2134/14034905.v1. 


\section{Finite-Time Relative Pose Tracking Control for Uncertain Spacecraft Rendezvous and Docking}

\author{
Liang Sun, Jinwei Wang \\ School of Automation and Electrical Engineering \\ University of Science and Technology Beijing \\ Beijing 100083, P. R. China \\ Email: liangsun@ustb.edu.cn; g20208728@xs.ustb.edu.cn
}

\author{
Jingjing Jiang \\ Department of Aeronautical and Automotive Engineering \\ Loughborough University \\ Loughborough, Leicestershire, LE11 3TU, UK \\ Email: J.Jiang2@lboro.ac.uk
}

\begin{abstract}
This study investigates the robust finite-time pose tracking control for spacecraft autonomous rendezvous and docking with parametric uncertainties and bounded external disturbances. Based on the uncertainly coupled relative pose dynamics, a fast terminal sliding mode controller is developed to achieve the finite-time convergence of the pose tracking errors. To reduce the control chattering results from the signum function in the controller, an exponential reaching law is employed to achieve the decreasing of the reaching time towards the sliding surface. The explicit tuning rules for designing parameters are derived based on the stability analysis of the closed-loop system. It is proved in Lyapunov framework that all closed-loop signals are always kept bounded and the pose tracking error converges to small neighborhood of zero in finite time. Simulation results validate the performance of the proposed robust finite-time control strategy.
\end{abstract}

\section{INTRODUCTION}

Autonomous rendezvous and docking control is an essential technology for the International Space Station supply and assembly of space systems. For the rendezvous and docking missions, satisfied performance and behavior of the control systems are the aim of the designing and manufacturing, such as high precision, strong robustness, and fast responses. These requirements are generally considered into the control system design to ensure the safe and reliable practical realization [1]. Thus the controller design for the rendezvous and docking under the requirement of satisfied controlled performance is always the hot topic in the control engineering field.

There are many linear controllers in previous decades for the linear relative orbital model, but they are unsuitable for the complicated and modern practical requirements for highperformance rendezvous and docking missions. Therefore, many advanced nonlinear controllers were proposed based on the nonlinear relative attitude and orbital dynamical mode in recent years, such as the adaptive output feedback controller [2], the line-of-sight based state feedback controllers [3], the model predictive controllers [4][5], the classical proportionalintegrative-derivative controller [6], state-dependent Riccati equation based nonlinear optimal controller [7], the modelbased backstepping controller [8] and sliding mode controller [9]. However, since the convergent time of the system states are generally prescribed by the designers in advance such that the controlled spacecraft system should have a finite- time controller to achieve the missions from the initial system states. Therefore, there are many finite-time controllers were designed for spacecraft systems in recent years, such as the trajectory planning-based robust adaptive integrated translation and rotation finite-time controller [10], the exponential coordinates description-based finite-time state feedback controller [11], the passivity-based adaptive finite-time controller [12], the finite-time extended state disturbance observer [13], and the nonlinear terminal sliding mode-based linear model predictive controller [14]. Then the collision avoidance and the maneuvering time constraints for spacecraft formation missions were included in the mathematical formulation of a linear programming problem in [15]. A coordinate-free finitetime attitude controller was developed in [16] to ensure that the required thrust vector is met exactly at the prescribed time. The integrated six-degrees of freedom relative pose finite-time controller was designed in [17] for spacecraft rendezvous with large-scale space structure. A finite-Time concurrent learning adaptive controller was proposed in [18] for spacecraft with inertia parameter identification without the persistent excitation required. In spite of solving the finite-time control problem for spacecraft missions in aforementioned studies, some of them ignored the couplings between relative translation and relative rotation motions, and some of them designed the finitetime controller without using the inherent passive properties of the relative dynamics such that the controller has complicated forms.

This study investigates the relative dynamics control problem of two spacecraft in rendezvous and docking missions, and the finite-time relative translational-rotational integrated controller was designed. In particular, the classical nonsingular terminal sliding mode technique and the exponential reaching law technique are combined in the simplified way to achieve the high performance of state convergence as long as the bounds of all model uncertainties are known. The finite-time convergence of the relative pose and velocity between two spacecraft is proved rigorously in the Lyapunov framework. The finite-time controller design and stability analysis approach can be easily extended to the general Euler-Lagrange mechanical systems based on their inherent passive properties. The theoretical analysis and the simulation example validate the effectiveness of the proposed controller. 
The rest of the paper is divided into four sections. Section II presents the mathematical description and control task for spacecraft rendezvous and proximity. Section III provides the concrete controller design results, and the stability of the closed-loop system is rigorously analyzed. Numerical simulations are conducted in Section IV to support the theoretic developments. Finally, conclusions are drawn in Section V.

\section{Problem Statement}

Consider the relative motion of a chaser spacecraft to a cooperative target spacecraft in a general Keplerian orbit. The Local-Vertical-Local-Horizontal(LVLH) frame with $(x, y, z)$ axes and origin fixed to the target spacecraft is used to describe the relative position motion. The $+z$ axis directs to the center of the Earth, the $+y$ axis points to the negative orbit normal, and the $+x$ axis is along the velocity vector of the target. The scalar $\bar{r}>0$ is the distance between the center of mass of target and center of the Earth, and $\rho=\sqrt{x^{2}+y^{2}+z^{2}}$ is the distance between the chaser's center of mass and the target's center of mass. It is assumed that distance between two spacecraft is small compared with the target's orbit radius, that is $\bar{r} \gg \rho$, and bounded disturbances and control forces are applied to the two spacecraft. These assumptions are generally made in the literature on the autonomous rendezvous and docking control for a short time period. Then, the relative motion equations can be described in LVLH frame as [19]:

$$
\left\{\begin{array}{l}
\ddot{x}-\ddot{\nu} z+2 \dot{\nu} \dot{z}-\left(\dot{\nu}^{2}-\frac{\mu}{\bar{r}^{3}}\right) x=\frac{f_{o 1}+d_{o 1}}{m} \\
\ddot{y}+\frac{\mu}{\bar{r}^{3}} y=\frac{f_{o 2}+d_{o 2}}{m} \\
\ddot{z}+\ddot{\nu} x+2 \dot{\nu} \dot{x}-\left(\dot{\nu}^{2}+2 \frac{\mu}{\bar{r}^{3}}\right) z=\frac{f_{o 3}+d_{o 3}}{m}
\end{array}\right.
$$

where $m$ is the mass of chaser; $\boldsymbol{f}_{o}=\left[f_{o 1}, f_{o 2}, f_{o 3}\right]^{\mathrm{T}}$ and $\boldsymbol{d}_{o}=\left[d_{o 1}, d_{o 2}, d_{o 3}\right]^{\mathrm{T}}$ are control and disturbance forces of the chaser spacecraft; $\mu=3.986 \times 10^{14}\left(\mathrm{~m}^{3} / \mathrm{s}^{2}\right)$ is the Earth gravitational coefficient; $\nu$ is the true anomaly of the target orbit. The evolution of $\bar{r}$ and $\nu$ is governed by [1]

$$
\begin{gathered}
\dot{\bar{r}}=-\frac{\bar{r} \ddot{\nu}}{2 \dot{\nu}}, \\
\dot{\nu}=\frac{n\left(1+e_{k} \cos \nu\right)^{2}}{\left(1-e_{k}^{2}\right)^{\frac{3}{2}}}, \\
\ddot{\nu}=\frac{-2 n^{2}\left(1+e_{k} \cos \nu\right)^{3} e_{k} \sin \nu}{\left(1-e_{k}^{2}\right)^{3}},
\end{gathered}
$$

where $e_{k}$ is the target orbital eccentricity, $n=\sqrt{\frac{\mu}{a_{k}^{3}}}$ is the target mean orbital angular velocity, $a_{k}=\frac{r_{k}}{1-e_{k}}$ is the target orbital semi-major axis, $r_{k}$ is the target perigee altitude.

The line-of-sight (LOS) frame with $\left(x_{s}, y_{s}, z_{s}\right)$ axis and origin located at the center of mass of the target is also used to describe the relative position motion as the LVLH frame. Its $x_{s}$-axis points from the center of target to the center of chaser, $z_{s}$-axis is vertical with $x_{s}$-axis in oxz plane of the LVLH frame, and finally the direction of $y_{s}$-axis completes a right handed orthogonal frame. Then, we use $\alpha \in(-\pi / 2, \pi / 2)$ to denote the elevation angle between the LOS and its projection in $o x z$ plane of the LVLH frame, and use $\beta \in(-\pi, \pi)$ to denote the azimuth angle between the $x$-axis of the LVLH frame and the LOS projection in oxz plane of the LVLH frame as shown in Figure 1.

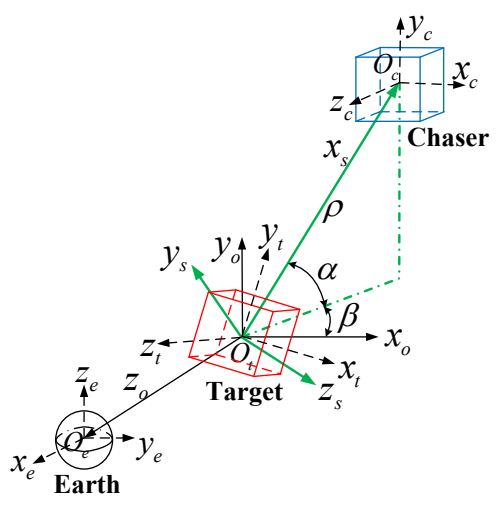

Fig. 1. Coordinate systems

Define a spherical coordinate vector $\boldsymbol{r}=[\rho, \alpha, \beta]^{\mathrm{T}}$ which satisfies

$$
x=\rho \cos \alpha \cos \beta, \quad y=\rho \sin \alpha, \quad z=-\rho \cos \alpha \sin \beta
$$

where $\rho$ is the distance between the center of mass of two spacecraft. With the transformation (2), the relative position motion equation (12) can be described in the LOS frame as [3]

$$
M_{1}(\boldsymbol{r}) \ddot{\boldsymbol{r}}+N_{1}(\boldsymbol{r}, \dot{\boldsymbol{r}}) \dot{\boldsymbol{r}}+\boldsymbol{g}_{1}(\boldsymbol{r})=E\left(\boldsymbol{f}_{s}+\boldsymbol{d}_{s}\right)
$$

where $M_{1}(\boldsymbol{r})=m H, N_{1}(\boldsymbol{r}, \dot{\boldsymbol{r}})=m A, \boldsymbol{g}_{1}(\boldsymbol{r})=m \boldsymbol{a}, H=$ $\operatorname{diag}\left\{1, \rho^{2}, \rho^{2} \cos ^{2} \alpha\right\}$,

$$
\begin{gathered}
A=\left[\begin{array}{ccc}
0 & -\rho \dot{\alpha} & -c_{1} \cos ^{2} \alpha \\
\rho \dot{\alpha} & \rho \dot{\rho} & c_{2} \sin \alpha \cos \alpha \\
c_{1} \cos ^{2} \alpha & -c_{2} \sin \alpha \cos \alpha & c_{3}
\end{array}\right], \\
\boldsymbol{a}=\left[\begin{array}{c}
-\rho\left[\dot{\nu}^{2} \cos ^{2} \alpha-\frac{\mu}{\bar{r}^{3}}\left(1-3 \cos ^{2} \alpha \sin ^{2} \beta\right]\right. \\
\rho^{2}\left[\dot{\nu}^{2} \cos \alpha \sin \alpha+\frac{\mu}{\bar{r}^{3}}\left(3 \sin ^{2} \beta \cos \alpha \sin \alpha\right)\right] \\
-\rho^{2}\left[\frac{\mu}{\bar{r}^{3}}\left(3 \cos ^{2} \alpha \sin \beta \cos \beta\right)-\ddot{\nu} \cos ^{2} \alpha\right]
\end{array}\right],
\end{gathered}
$$

$c_{1}=\rho \dot{\beta}-2 \rho \dot{\nu}, c_{2}=\rho^{2} \dot{\beta}-2 \rho^{2} \dot{\nu}, c_{3}=\rho \dot{\rho} \cos ^{2} \alpha-$ $\rho^{2} \dot{\alpha} \sin \alpha \cos \alpha ; E=\operatorname{diag}\{1, \rho,-\rho \cos \alpha\} ; \boldsymbol{f}_{s}=R_{s o} \boldsymbol{f}_{o}$ and $\boldsymbol{d}_{s}=R_{s o} \boldsymbol{d}_{o}$ are control and disturbance forces represented in the LOS frame; $R_{s o}$ is the rotational matrix from the LVLH frame to the LOS frame and

$$
R_{s o}=\left[\begin{array}{ccc}
\cos \alpha \cos \beta & -\sin \alpha \cos \beta & \sin \beta \\
\sin \alpha & \cos \alpha & 0 \\
-\cos \alpha \sin \beta & \sin \alpha \sin \beta & \cos \beta
\end{array}\right] .
$$

Suppose the chaser is a rigid body, and the orientation of a rigid spacecraft with respect to the Earth-Centered Inertial (ECI) frame can be described by MRPs as

$$
\boldsymbol{\sigma} \triangleq \zeta \tan \left(\frac{\vartheta}{4}\right)
$$


where $\vartheta \in(-2 \pi, 2 \pi)$ is the rotation angle about the Euler axis, and $\zeta$ is the unit vector of the Euler axis. As stated in [19], the shadow MRP set can be exploited to yield a globally nonsingular attitude description with a minimal threeparameter coordinate set at the expense of a discontinuity. To avoid the singularity, the MRP set is switched to the shadow set before reaching the singularity. A convenient switching condition is the unit magnitude surface $\|\boldsymbol{\sigma}\|=1$, such that the composite MRP description always satisfies $\|\boldsymbol{\sigma}\| \leq 1$. This surface represents all possible orientations in which the body has performed a principal rotation relative the origin $\vartheta=\pi$. Note that on this surface there are two possible MRP sets that describe the same attitude. Then the chaser attitude dynamics in terms of the MRPs take in the form [19]

$$
\left\{\begin{array}{l}
\dot{\boldsymbol{\sigma}}=G(\boldsymbol{\sigma}) \boldsymbol{\omega} \\
J \dot{\boldsymbol{\omega}}+S(\boldsymbol{\omega}) J \boldsymbol{\omega}=\tau_{u}+\boldsymbol{\tau}_{w}
\end{array}\right.
$$

with $G(\boldsymbol{\sigma})=\frac{1}{4}\left[\left(1-\boldsymbol{\sigma}^{\mathrm{T}} \boldsymbol{\sigma}\right) I_{3}+2 S(\boldsymbol{\sigma})+2 \boldsymbol{\sigma} \boldsymbol{\sigma}^{\mathrm{T}}\right]$, where $\boldsymbol{\sigma} \in \mathbb{R}^{3}$ and $\boldsymbol{\omega} \in \mathbb{R}^{3}$ are the chaser attitude and angular velocity with respect to the ECI frame, respectively; $J$ is the inertial matrix of the chaser; $\boldsymbol{\tau}_{u}$ and $\boldsymbol{w}_{u}$ are control and disturbance torques. These are all represented in the chaser's body-fixed frame. Moreover, $I_{3}$ is the $3 \times 3$ identity matrix. The rotation matrix from the ECI frame to the chaser's body-fixed frame is defined as

$$
R_{c i}=I_{3}-\frac{4\left(1-\boldsymbol{\sigma}^{\mathrm{T}} \boldsymbol{\sigma}\right)}{\left(1+\boldsymbol{\sigma}^{\mathrm{T}} \boldsymbol{\sigma}\right)^{2}} S(\boldsymbol{\sigma})+\frac{8}{\left(1+\boldsymbol{\sigma}^{\mathrm{T}} \boldsymbol{\sigma}\right)^{2}} S^{2}(\boldsymbol{\sigma})
$$

and $S(\boldsymbol{\xi})$ denotes a skew-symmetric matrix which is given by

$$
S(\boldsymbol{\xi}) \triangleq\left[\begin{array}{ccc}
0 & -\xi_{3} & \xi_{2} \\
\xi_{3} & 0 & -\xi_{1} \\
-\xi_{2} & \xi_{1} & 0
\end{array}\right]
$$

and satisfies $\boldsymbol{\xi}^{\mathrm{T}} S(\boldsymbol{\xi})=0, S(\boldsymbol{\xi}) \boldsymbol{\eta}=-S(\boldsymbol{\eta}) \boldsymbol{\xi}$, and $\boldsymbol{\xi}^{\mathrm{T}} S(\boldsymbol{\eta}) \boldsymbol{\xi}=$ 0 for any vectors $\boldsymbol{\xi}=\left[\xi_{1}, \xi_{2}, \xi_{3}\right]^{\mathrm{T}}$ and $\boldsymbol{\eta} \in \mathbb{R}^{3}$.

As stated in [19], one has $G^{-1}(\boldsymbol{\sigma})=\frac{16}{\left(1+\boldsymbol{\sigma}^{\mathrm{T}} \boldsymbol{\sigma}\right)^{2}} G^{\mathrm{T}}(\boldsymbol{\sigma})$. Denote $P=G^{-1}(\boldsymbol{\sigma})$, the chaser's attitude motion equation (4) can be rewritten as

$$
M_{2}(\boldsymbol{\sigma}) \ddot{\boldsymbol{\sigma}}+N_{2}(\boldsymbol{\sigma}, \dot{\boldsymbol{\sigma}}) \dot{\boldsymbol{\sigma}}=P^{\mathrm{T}}\left(\boldsymbol{\tau}_{u}+\boldsymbol{\tau}_{w}\right)
$$

where $M_{2}(\boldsymbol{\sigma})=P^{\mathrm{T}} J P, N_{2}(\boldsymbol{\sigma}, \dot{\boldsymbol{\sigma}})=P^{\mathrm{T}} J \dot{P}-P^{\mathrm{T}} S(J P \dot{\boldsymbol{\sigma}}) P$.

Thus, recalling (3) and (5), and denoting system state $\boldsymbol{q}=$ $\left[\boldsymbol{r}^{\mathrm{T}}, \boldsymbol{\sigma}^{\mathrm{T}}\right]^{\mathrm{T}}$, the integrated dynamics of relative position and attitude between two spacecraft can be derived as

$$
\mathcal{M}(\boldsymbol{q}) \ddot{\boldsymbol{q}}+\mathcal{N}(\boldsymbol{q}, \dot{\boldsymbol{q}}) \dot{\boldsymbol{q}}+\boldsymbol{g}(\boldsymbol{q})=R(\boldsymbol{u}+\boldsymbol{d})
$$

where $\mathcal{M}(\boldsymbol{q})=\operatorname{diag}\left\{M_{1}(\boldsymbol{r}), M_{2}(\boldsymbol{\sigma})\right\}, R=\operatorname{diag}\left\{E R_{s c}, P^{\mathrm{T}}\right\}$, $\mathcal{N}(\boldsymbol{q}, \dot{\boldsymbol{q}})=\operatorname{diag}\left\{N_{1}(\boldsymbol{r}, \dot{\boldsymbol{r}}), N_{2}(\boldsymbol{\sigma}, \dot{\boldsymbol{\sigma}})\right\}, \boldsymbol{g}(\boldsymbol{q})=\left[\boldsymbol{g}_{1}^{\mathrm{T}}(\boldsymbol{r}), \mathbf{0}\right]^{\mathrm{T}}$, $\boldsymbol{u}=\left[\boldsymbol{f}_{c}^{\mathrm{T}}, \boldsymbol{\tau}_{u}^{\mathrm{T}}\right]^{\mathrm{T}}, \boldsymbol{d}=\left[\boldsymbol{d}_{c}^{\mathrm{T}}, \boldsymbol{\tau}_{w}^{\mathrm{T}}\right]^{\mathrm{T}}, \boldsymbol{f}_{c}$ and $\boldsymbol{d}_{c}$ are control and disturbance forces of the chaser expressed in the chaser's body-fixed frame, respectively. $R_{s c}=R_{s i} R_{c i}^{\mathrm{T}}$ is the coordinate transformation matrix from the chaser's body-fixed frame to the LVLH frame, $R_{s i}=R_{s o} R_{o i}$, and $R_{o i}$ is the rotation matrix from the ECI frame to the LVLH frame and can be computed by the target orbital elements as follows

$$
R_{o i}=\left[\begin{array}{ccc}
\mathrm{c}_{1} \mathrm{c}_{3}-\mathrm{s}_{1} \mathrm{c}_{2} \mathrm{~s}_{3} & \mathrm{c}_{1} \mathrm{~s}_{3}+\mathrm{s}_{1} \mathrm{c}_{2} \mathrm{c}_{3} & \mathrm{~s}_{1} \mathrm{~s}_{2} \\
\mathrm{~s}_{1} \mathrm{c}_{3}-\mathrm{c}_{1} \mathrm{c}_{2} \mathrm{~s}_{3} & \mathrm{~s}_{1} \mathrm{~s}_{3}+\mathrm{c}_{1} \mathrm{c}_{2} \mathrm{c}_{3} & \mathrm{c}_{1} \mathrm{~s}_{2} \\
\mathrm{~s}_{2} \mathrm{~s}_{3} & -\mathrm{s}_{2} \mathrm{c}_{3} & \mathrm{c}_{2}
\end{array}\right],
$$

where $\mathrm{c}_{i} \triangleq \cos \gamma_{i}, \mathrm{~s}_{i} \triangleq \sin \gamma_{i}(i=1,2,3)$, and $\gamma_{1}$ is right ascension of ascending node, $\gamma_{2}$ is inclination, $\gamma_{3}$ is argument of perigee.

The matrices $\mathcal{M}(\boldsymbol{q}), \mathcal{N}(\boldsymbol{q}, \dot{\boldsymbol{q}})$ and $\boldsymbol{g}(\boldsymbol{q})$ in (6) satisfy the following relationships

$$
\begin{gathered}
\lambda_{m} \leq\|\mathcal{M}(\boldsymbol{q})\| \leq \lambda_{M} \\
\boldsymbol{\zeta}^{\mathrm{T}}[\dot{\mathcal{M}}(\boldsymbol{q})-2 \mathcal{N}(\boldsymbol{q}, \dot{\boldsymbol{q}})] \boldsymbol{\zeta}=0
\end{gathered}
$$

where $\lambda_{m}>0$ and $\lambda_{M}>0$ are minimum and maximum eigenvalues of matrix $\mathcal{M}(\boldsymbol{q})$, and $\boldsymbol{\zeta} \in \mathbb{R}^{6}$.

In this paper, following assumptions are exploited in the subsequent development.

Assumption 1: In practice, the true values of the inertia parameters are difficult or even impossible to obtain because of the measurement errors or disturbances, thus it is assumed that $m=m_{0}+m_{\Delta}$ and $J=J_{0}+J_{\Delta}$, where $m_{0}$ and $J_{0}$ are nominal values; $m_{\Delta}$ and $J_{\Delta}$ are uncertainties and bounded by $\left|m_{\Delta}\right| \leq \bar{m}_{\Delta}$ and $\left\|J_{\Delta}\right\| \leq \bar{J}_{\Delta}$ with two known constants $\bar{m}_{\Delta}$ and $\bar{J}_{\Delta}$, respectively. The external disturbance $\boldsymbol{d}$ is bounded by $\|\boldsymbol{d}\| \leq \bar{d}$ with known constant $\bar{d}$.

Assumption 2: The position $\boldsymbol{q}$ and velocity $\dot{\boldsymbol{q}}$ are both measurable. The chaser's docking port is toward the direction of its body-fixed frame $+x$ axis, and the target's docking port is toward the direction of its body-fixed frame $-x$ axis. The cooperative target's attitude is stabilized in the LVLH frame, and the chaser's attitude motion only requires to track the target's attitude trajectory.

Under Assumption 2, we know that desired attitude trajectory of the chaser is the target's attitude trajectory. Therefore, the autonomous rendezvous and docking control objective is to drive the chaser at a certain safe distance from the target while keep its docking port facing the docking port of the target. The attitude trajectory of two spacecraft must be kept the same during the chaser's maneuver so that subsequent operations, for instance capturing or docking, can be carried out safely. Therefore, by defining desired trajectory $\boldsymbol{q}_{t}(t)=$ $\left[\boldsymbol{r}_{t}^{\mathrm{T}}(t), \boldsymbol{\sigma}_{t}^{\mathrm{T}}(t)\right]^{\mathrm{T}}$ with desired relative position $\boldsymbol{r}_{t}(t) \in \mathbb{R}^{3}$ and desired attitude $\boldsymbol{\sigma}_{t}(t) \in \mathbb{R}^{3}$, we conclude that the control objective is to ensure that the system tracks a desired trajectory $\boldsymbol{q}_{t}(t) \in \mathbb{R}^{6}$ despite control input constraints and unavailable velocities. To quantify the tracking objective, the tracking errors can be defined by

$$
\boldsymbol{e}(t)=\boldsymbol{q}(t)-\boldsymbol{q}_{t}(t), \dot{\boldsymbol{e}}(t)=\dot{\boldsymbol{q}}(t)-\dot{\boldsymbol{q}}_{t}(t)
$$

where desired trajectory satisfies $\left\|\left[\boldsymbol{q}_{t}^{\mathrm{T}}(t), \dot{\boldsymbol{q}}_{t}^{\mathrm{T}}(t), \ddot{\boldsymbol{q}}_{t}^{\mathrm{T}}(t)\right]^{\mathrm{T}}\right\| \leq$ $\lambda_{t}$ with a known constant $\lambda_{t}$. Thus, the control objective is equivalent to synthesize a bounded control input $\boldsymbol{u}$ such that tracking error $\boldsymbol{e}(t)$ converges to small neighborhood of zero in finite time. 


\section{Controller Design And Stability AnAlysis}

Lemma 1: Suppose $V(x)$ is a continuously differentiable positive definite function defined in a neighborhood $U \subset \mathbb{R}^{n}$ of the origin, and there exist constants $\lambda>0$ and $0<\mu<1$ such that $\dot{V}(x)+\lambda V^{\mu}(x)$ is a negative semidefinite $U$, then $V(x) \equiv 0$ can be reached in finite time. Moreover, the settlingtime $T_{s}$ satisfies

$$
T_{s} \leq \frac{V^{1-\mu}\left(x_{0}\right)}{\lambda(1-\mu)}
$$

Lemma 2: For any real number $l_{i}, i=1, \cdots, n$, if $0<$ $\zeta_{1}<1$ and $0<\zeta_{2}<2$, then the following inequalities hold:

$$
\begin{gathered}
\left(\left|l_{1}\right|+\left|l_{2}\right|+\cdots+\left|l_{n}\right|\right)^{\zeta_{1}} \leq\left|l_{1}\right|^{\zeta_{1}}+\left|l_{2}\right|^{\zeta_{1}}+\cdots+\left|l_{n}\right|^{\zeta_{1}} \\
\left(\left|l_{1}\right|^{2}+\left|l_{2}\right|^{2}+\cdots+\left|l_{n}\right|^{2}\right)^{\zeta_{2}} \leq\left(\left|l_{1}\right|^{\zeta_{2}}+\left|l_{2}\right|^{\zeta_{2}}+\cdots+\left|l_{n}\right|^{\zeta_{2}}\right)^{2}
\end{gathered}
$$

Letting $\boldsymbol{x}_{1}=\boldsymbol{e}$ and $\boldsymbol{x}_{2}=\dot{\boldsymbol{x}}_{1}=\dot{\boldsymbol{e}}$, it follows that $\boldsymbol{q}=$ $\boldsymbol{x}_{1}+\boldsymbol{q}_{t}$ and $\dot{\boldsymbol{q}}=\boldsymbol{x}_{2}+\dot{\boldsymbol{q}}_{t}$, so (6) can be rewritten as

$$
\left\{\begin{array}{l}
\dot{\boldsymbol{x}}_{1}=\boldsymbol{x}_{2} \\
\mathcal{M} \dot{\boldsymbol{x}}_{2}+\mathcal{N} \boldsymbol{x}_{2}=R \boldsymbol{u}-\mathcal{M}_{0} \ddot{\boldsymbol{q}}_{t}-\mathcal{N}_{0} \dot{\boldsymbol{q}}_{t}-\boldsymbol{g}_{0}+\boldsymbol{w}
\end{array}\right.
$$

where $\boldsymbol{w}=R \boldsymbol{d}-\mathcal{M}_{\Delta} \ddot{\boldsymbol{q}}_{t}-\mathcal{N}_{\Delta} \dot{\boldsymbol{q}}_{t}-\boldsymbol{g}_{\Delta}, \mathcal{M}_{0}=$ $\operatorname{diag}\left\{M_{10}, M_{20}\right\}, \mathcal{M}_{\Delta}=\operatorname{diag}\left\{M_{1 \Delta}, M_{2 \Delta}\right\}, \mathcal{N}_{0}=$ $\operatorname{diag}\left\{N_{10}, N_{20}\right\}, \mathcal{N}_{\Delta}=\operatorname{diag}\left\{N_{1 \Delta}, N_{2 \Delta}\right\}, \boldsymbol{g}_{0}=\left[\boldsymbol{g}_{10}^{\mathrm{T}}(\boldsymbol{r}), \mathbf{0}\right]^{\mathrm{T}}$, $\boldsymbol{g}_{\Delta}=\left[\boldsymbol{g}_{1 \Delta}^{\mathrm{T}}(\boldsymbol{r}), \mathbf{0}\right]^{\mathrm{T}}, M_{10}=m_{0} H, N_{10}=m_{0} A, \boldsymbol{g}_{10}=m_{0} \boldsymbol{a}$, $M_{1 \Delta}=m_{\Delta} H, N_{1 \Delta}=m_{\Delta} A, \boldsymbol{g}_{1 \Delta}=m_{\Delta} \boldsymbol{a}, M_{20}=P^{\mathrm{T}} J_{0} P$, $N_{20}=P^{\mathrm{T}} J_{0} \dot{P}-P^{\mathrm{T}} S\left(J_{0} P \dot{\boldsymbol{\sigma}}\right) P, M_{2 \Delta}=P^{\mathrm{T}} J_{\Delta} P, N_{2 \Delta}=$ $P^{\mathrm{T}} J_{\Delta} \dot{P}-P^{\mathrm{T}} S\left(J_{\Delta} P \dot{\boldsymbol{\sigma}}\right) P$.

Consider the uncertain nonlinear dynamics in (10). Define terminal sliding mode variable

$$
s=\boldsymbol{x}_{2}-\phi_{1}\left(\boldsymbol{x}_{1}\right)
$$

where $\phi_{1}\left(\boldsymbol{x}_{1}\right)$ is a auxiliary function with $\phi_{1}(\mathbf{0})=\mathbf{0}$. Then, substituting (11) into (10) yields

$$
\left\{\begin{array}{l}
\dot{\boldsymbol{x}}_{1}=\boldsymbol{s}+\boldsymbol{\phi}_{1} \\
\mathcal{M} \dot{\boldsymbol{s}}+\mathcal{N} \boldsymbol{s}=R \boldsymbol{u}-\mathcal{M}_{0}\left(\ddot{\boldsymbol{q}}_{t}+\dot{\boldsymbol{\phi}}_{1}\right)-\mathcal{N}_{0}\left(\dot{\boldsymbol{q}}_{t}+\boldsymbol{\phi}_{1}\right)-\boldsymbol{g}_{0}+\boldsymbol{\delta}
\end{array}\right.
$$

where $\boldsymbol{\delta}=\boldsymbol{w}-\mathcal{M}_{\Delta} \dot{\phi}_{1}-\mathcal{N}_{\Delta} \phi_{1}$.

To achieve finite-time convergence of system states, the following two auxiliary functions are defined by

$$
\left\{\begin{array}{l}
\phi_{1}\left(\boldsymbol{x}_{1}\right)=-K_{1} \operatorname{sig}\left(\boldsymbol{x}_{1}\right)^{\gamma} \\
\boldsymbol{\phi}_{2}(\boldsymbol{s})=-K_{2} \operatorname{sig}(\boldsymbol{s})^{\gamma}
\end{array}\right.
$$

where $K_{i}=\operatorname{diag}\left\{k_{i 1}, k_{i 2}, \cdots, k_{i 6}\right\}(i=1,2)$ are positive definite gain matrices, $\operatorname{sig}(\boldsymbol{x})^{\gamma} \triangleq$ $\left[\left|x_{1}\right|^{\gamma} \operatorname{sign}\left(x_{1}\right),\left|x_{2}\right|^{\gamma} \operatorname{sign}\left(x_{2}\right), \cdots,\left|x_{n}\right|{ }^{\gamma} \operatorname{sign}\left(x_{n}\right)\right]^{\mathrm{T}}$ for any $\boldsymbol{x}=\left[x_{1}, x_{2}, \cdots, x_{n}\right]^{\mathrm{T}}, 0<\gamma<1$, and $\operatorname{sign}\left(x_{i}\right)$ for any $x_{i}(i=1,2, \cdots, n)$ is the signum function, which is defined as follows:

$$
\operatorname{sign}\left(x_{i}\right)= \begin{cases}1 & x_{i}>0 \\ 0 & x_{i}=0 \\ -1 & x_{i}<0\end{cases}
$$

Theorem 1: Consider the system (12) under Assumptions 1 and 2. Design the robust finite-time control law as

$$
\begin{aligned}
\boldsymbol{u}= & R^{-1}\left[\mathcal{M}_{0}\left(\ddot{\boldsymbol{q}}_{t}+\dot{\boldsymbol{\phi}}_{1}\right)+\mathcal{N}_{0}\left(\dot{\boldsymbol{q}}_{t}+\boldsymbol{\phi}_{1}\right)\right. \\
& \left.+\boldsymbol{g}_{0}+\boldsymbol{\phi}_{2}-\boldsymbol{x}_{1}-\bar{\delta} \operatorname{sign}(\boldsymbol{s})\right]
\end{aligned}
$$

where $\bar{\delta}=\delta_{a} / \delta_{b}, \quad \delta_{a}=\bar{d}\|R\|+\left(\bar{m}_{\Delta}\|H\|+\right.$ $\left.\bar{J}_{\Delta}\left\|P^{\mathrm{T}}\right\|\|P\|\right)\left(\left\|\dot{\phi}_{1}\right\|+\lambda_{t}\right)+\left(\bar{m}_{\Delta}\|A\|+\bar{J}_{\Delta}\left\|P^{\mathrm{T}}\right\|\|\dot{P}\|+\right.$ $\left.\bar{J}_{\Delta}\left\|P^{\mathrm{T}}\right\|\|P\|^{2}\|\dot{\boldsymbol{\sigma}}\|\right)\left(\left\|\phi_{1}\right\|+\lambda_{t}\right)+\bar{m}_{\Delta}\|a\|, \delta_{b}=\delta_{c}+(1-$ $\left.\delta_{c}\right) \mathrm{e}^{-\delta_{0}\|\boldsymbol{s}\|^{p}}, 0<\delta_{c}<1, \delta_{0}>0, p>0$, then the proposed controller (14) can guarantee the system states $e$ and $\dot{e}$ converge to zero in finite time.

Proof. Substituting controller (14) to (12) yields the closedloop system

$$
\left\{\begin{array}{l}
\dot{x}_{1}=s+\phi_{1} \\
\mathcal{M} \dot{s}+\mathcal{N} s=\phi_{2}-x_{1}-\bar{\delta} \operatorname{sign}(s)+\delta
\end{array}\right.
$$

Define a positive-definite Lyapunov function

$$
V=\frac{1}{2}\left(\boldsymbol{x}_{1}^{\mathrm{T}} \boldsymbol{x}_{1}+\boldsymbol{s}^{\mathrm{T}} \mathcal{M} \boldsymbol{s}\right)
$$

Taking time derivative of $V$ along the state trajectory of the closed-loop system (15), and using the facts $s^{\mathrm{T}}(\dot{\mathcal{M}}-2 \mathcal{N}) s=$ 0 from (8) and $\boldsymbol{s}^{\mathrm{T}} \boldsymbol{\delta}-\boldsymbol{s}^{\mathrm{T}} \bar{\delta} \operatorname{sign}(\boldsymbol{s}) \leq 0$ from $\|\boldsymbol{\delta}\| \leq \bar{\delta}$, it follows that

$$
\begin{aligned}
\dot{V} & =\boldsymbol{x}_{1}^{\mathrm{T}} \dot{\boldsymbol{x}}_{1}+\boldsymbol{s}^{\mathrm{T}} \mathcal{M} \dot{\boldsymbol{s}}+\frac{1}{2} \boldsymbol{s}^{\mathrm{T}} \dot{\mathcal{M}} \boldsymbol{s} \\
& =\boldsymbol{x}_{1}^{\mathrm{T}} \boldsymbol{\phi}_{1}+\boldsymbol{s}^{\mathrm{T}} \boldsymbol{\phi}_{2}+\boldsymbol{s}^{\mathrm{T}} \boldsymbol{\delta}-\boldsymbol{s}^{\mathrm{T}} \bar{\delta} \operatorname{sign}(\boldsymbol{s}) \\
& \leq-\boldsymbol{x}_{1}^{\mathrm{T}} K_{1} \operatorname{sig}\left(\boldsymbol{x}_{1}\right)^{\gamma}-\boldsymbol{s}^{\mathrm{T}} K_{2} \operatorname{sig}(\boldsymbol{s})^{\gamma}
\end{aligned}
$$

Define $\mu=\frac{1+\gamma}{2}, \frac{1}{2}<\mu<1, k_{1 m}=\min \left\{k_{1 i}\right\}, k_{2 m}=$ $\min \left\{k_{2 i}\right\}, \bar{k}_{1}=2^{\mu} k_{1 m}, \bar{k}_{2}=2^{\mu} k_{2 m}, \bar{k}=\min \left\{\bar{k}_{1}, \frac{\bar{k}_{2}}{\lambda_{M}}\right\}$. Then, from Lemma 2, we have

$$
\begin{aligned}
\dot{V} & \leq-k_{1 m} \sum_{i=1}^{6}\left|x_{1 i}\right|^{1+\gamma}-k_{2 m} \sum_{i=1}^{6}\left|s_{i}\right|^{1+\gamma} \\
& \leq-k_{1 m}\left(\sum_{i=1}^{6} x_{1 i}^{2}\right)^{\frac{1+\gamma}{2}}-k_{2 m}\left(\sum_{i=1}^{6} s_{i}^{2}\right)^{\frac{1+\gamma}{2}} \\
& \leq-\bar{k}_{1}\left(\frac{1}{2} \sum_{i=1}^{6} x_{1 i}^{2}\right)^{\mu}-\bar{k}_{2}\left(\frac{1}{2} \sum_{i=1}^{6} s_{i}^{2}\right)^{\mu} \\
& \leq-\bar{k} V^{\mu}
\end{aligned}
$$

Thus, from Lemma 1, we know that the origin of the closedloop system is finite-time stable. This means that $\boldsymbol{x}_{1}$ and $s$ convergent to zero in finite time, then the system errors $e$ and $\dot{e}$ convergent to zero in finite time. From Lemma 1, we also know that the settling time satisfies $T_{s} \leq \frac{\bar{V}(0)^{1-\mu}}{\bar{k}(1-\mu)}$, where $\bar{V}(0)=\frac{1}{2}\left(\left\|\boldsymbol{x}_{1}(0)\right\|^{2}+\lambda_{M}\|\boldsymbol{s}(0)\|^{2}\right)$. Therefore, the closedloop system (15) with controller (14) provides high control precision, fast response performance, and strong robustness.

Remark 1: The time derivative of function $\operatorname{sig}\left(\boldsymbol{x}_{1}\right)$ in $\phi_{1}\left(\boldsymbol{x}_{1}\right)$ will be infinite when $x_{1 i}=0$ and $\dot{x}_{1 i} \neq 0$ in $\dot{\phi}_{1}\left(\boldsymbol{x}_{1}\right)$. To avoid computational singularity, the definition of $\dot{\phi}_{1}\left(\boldsymbol{x}_{1}\right)$ is modified as follows:

$$
\dot{\phi}_{1}\left(x_{1 i}\right)= \begin{cases}-k_{1 i} \gamma\left|x_{1 i}\right|^{\gamma-1} \dot{x}_{1 i} & \text { if }\left|x_{1 i}\right| \geq \epsilon \text { and } \dot{x}_{1 i} \neq 0 \\ -k_{1 i} \gamma\left|\kappa_{i}\right|^{\gamma-1} \dot{x}_{1 i} & \text { if }\left|x_{1 i}\right|<\epsilon \text { and } \dot{x}_{1 i} \neq 0 \\ 0 & \text { if } \dot{x}_{1 i}=0\end{cases}
$$

where $\epsilon$ and $\kappa_{i}$ are small positive constants, $x_{1 i}$ is the $i$ th entry of the vector $\boldsymbol{x}_{1}$, and $\dot{\phi}_{1}\left(x_{1 i}\right)$ is the $i$ th entry of the vector $\dot{\phi}_{1}\left(\boldsymbol{x}_{1}\right)$. Moreover, the parameter $\gamma$ should be chosen 
such that $0.7<\gamma<0.9$, because small $\gamma$ may result in undesired control chattering, which may excite unmodeled high-frequency dynamics.

Remark 2: Since $\delta_{b}$ is always strictly positive, then the exponential reaching law does not affect the stability of the closed-loop system. From the sliding mode surface, one can see that if $\|\boldsymbol{s}\|$ increases, $\delta_{b}$ approaches $\delta_{c}$, and then $\delta_{a} / \delta_{b}$ converges to $\delta_{a} / \delta_{c}$, which is larger than $\delta_{a}$. This implies that $\delta_{a} / \delta_{b}$ increases as $\|\boldsymbol{s}\|$ increases, then the convergence rate to $s=\mathbf{0}$ will be faster. Moreover, if $\|s\|$ decreases, then $\delta_{b}$ approaches one, and $\delta_{a} / \delta_{b}$ converges to $\delta_{a}$. This implies that, if the system errors approach to zero, $\delta_{a} / \delta_{b}$ gradually decreases to reduce the control effort. Therefore, the exponential reaching law permits the control law to dynamically adapt to the variations of $\|s\|$ by making $\delta_{a} / \delta_{b}$ to change between $\delta_{a}$ and $\delta_{a} / \delta_{c}$. If $\delta_{c}=1$, then the exponential reaching law becomes the same as the classical reaching law [21][22].

Remark 3: To avoid the undesired control chattering, the signum function $\operatorname{sign}(s)$ in (14) can be replaced by the hyperbolic tangent function $\tanh (\varsigma s)=\left[\tanh \left(\varsigma s_{1}\right), \cdots, \tanh \left(\varsigma s_{6}\right)\right]^{\mathrm{T}}$ with $\varsigma>0$. Then, according to the inequality $0 \leq\left|s_{i}\right|-$ $s_{i} \tanh \left(\varsigma s_{i}\right) \leq \frac{\iota}{\varsigma}$ with $\iota=0.2785$, we have $\dot{V}+\bar{k} V^{\mu} \leq \varepsilon$ with $\varepsilon=\frac{6 \bar{\delta} \iota}{\varsigma}$, thus the system states $e$ and $\dot{e}$ converge to $\varepsilon$-neighborhood of zero in finite time.

\section{Simulation Results and Analysis}

In this section, the simulation scenario describes an example of the cooperative spacecraft autonomous in-orbit rendezvous and docking mission, where the target has stabilized at the given attitude in the LVLH frame. The target is assumed to be in a low earth orbit with $r_{k}=400(\mathrm{~km})$ perigee altitude. Eccentricity $e_{k}=0.1375$. Orbit inclination is set to $\gamma_{2}=45^{\circ}$. Argument of perigee and right ascension of ascending node are all set to $\gamma_{1}=\gamma_{3}=0^{\circ}$.

We know the nominal value and the bound of uncertain part of the mass and inertia matrix are $m_{0}=20$, $J_{0}=\operatorname{diag}\{20,20,20\}, \bar{m}_{\Delta}=3$, and $\bar{J}_{\Delta}=2$, respectively. Moreover, the bound of external disturbances is $\bar{d}=0.03$. The simulation aim to control system state from $\boldsymbol{q}(0)=$ $[50, \pi / 6,-\pi / 6,0,0,0]^{\mathrm{T}}$ and $\dot{\boldsymbol{q}}(0)=[0.5,0.05,0.05,0,0,0]^{\mathrm{T}}$ to $\boldsymbol{q}_{t}=[5,0,0,0.3,0.4,0.3]^{\mathrm{T}}$ and $\dot{\boldsymbol{q}}_{t}=[0,0,0,0,0,0]^{\mathrm{T}}$. Thus, the bound of the desired trajectory can be set as $\lambda_{t}=6$. The proposed controller (14) is used to achieve the simulation objective under sampling time $0.1(\mathrm{~s})$, where the signum function in (14) is replaced by hyperbolic tangent function to avoid control chattering phenomenon. Controller parameters are set as $K_{1}=\operatorname{diag}\{0.02,0.02,0.04,0.2,0.2,0.2\}$, $K_{2}=\operatorname{diag}\{50,200,200,100,100,100\}, \gamma=0.9, \epsilon=0.1$, $\delta_{c}=\delta_{0}=0.01, p=0.9, \varsigma=0.002$.

Suppose the simulation parameters of the chaser are

$$
m=22(\mathrm{~kg}), \quad J=\left[\begin{array}{ccc}
21 & 0.1 & 0.2 \\
0.1 & 20 & 0.1 \\
0.2 & 0.1 & 20
\end{array}\right]\left(\mathrm{kgm}^{2}\right),
$$

$$
\begin{gathered}
\boldsymbol{d}_{c}=\left[\begin{array}{l}
1+3 \sin (n t)+3 \sin (n t) \\
2+2 \sin (n t)+3 \sin (n t) \\
1+3 \cos (n t)+2 \cos (n t)
\end{array}\right] \times 10^{-4}(\mathrm{~N}), \\
\boldsymbol{\tau}_{d}=\left[\begin{array}{l}
2+2 \sin (n t)+3 \sin (n t) \\
1+3 \sin (n t)+2 \sin (n t) \\
2+3 \cos (n t)+2 \cos (n t)
\end{array}\right] \times 10^{-5}(\mathrm{Nm}),
\end{gathered}
$$

where $n=\sqrt{\frac{\mu_{g}}{a_{k}^{3}}}, \mu=3.986 \times 10^{14}$ is gravitational constant of the Earth, $a_{k}=\frac{r_{k}}{1-e_{k}}$.

Simulation results are presented in Figures 2-5. Figure 2 and 3 show the time histories of the relative pose and velocities for translational motion, while Figure 4 shows the time histories of relative attitude and angular velocity for rotational motion, and Figure 5 shows the time histories of the control acceleration and the torque. It is seen that the relative pose and velocity ultimately converge to zero in finite time, and the relative position tracking and attitude synchronization can be achieved based on the proposed controller (14).
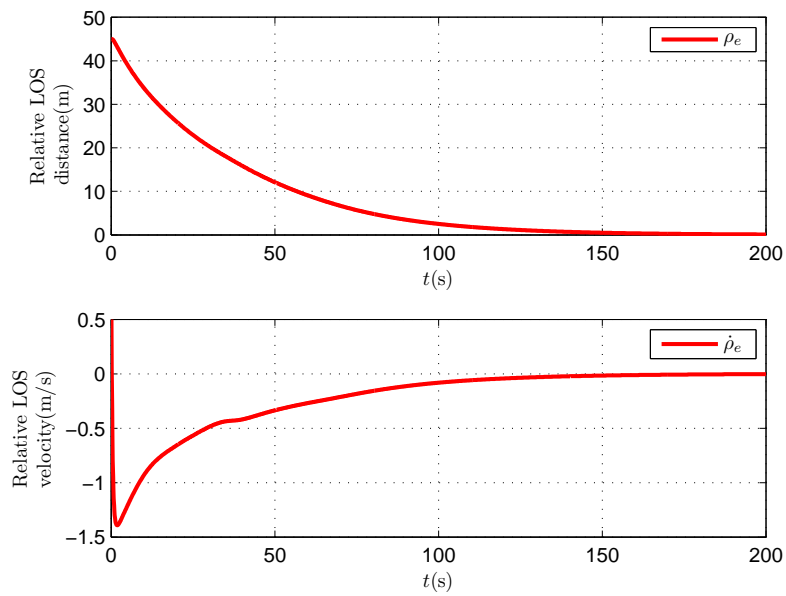

Fig. 2. Relative distance and velocity under controller (14).
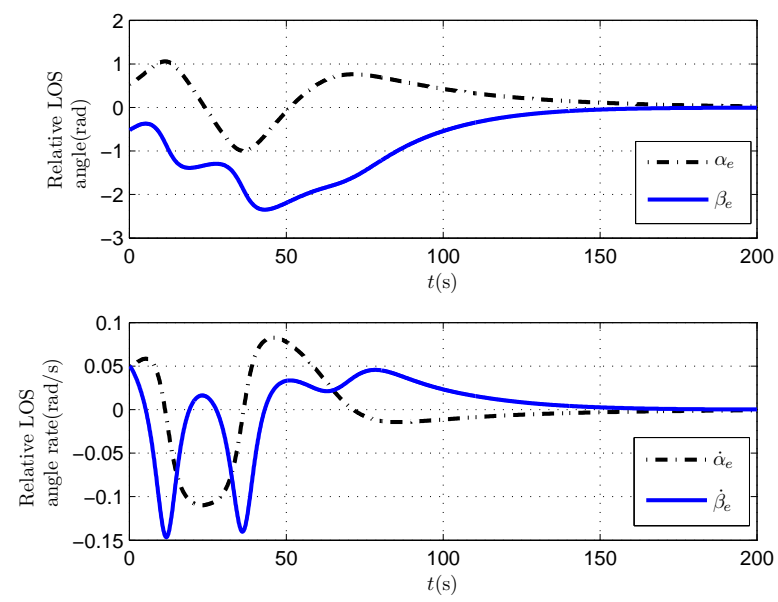

Fig. 3. Relative azimuth and velocity under controller (14). 

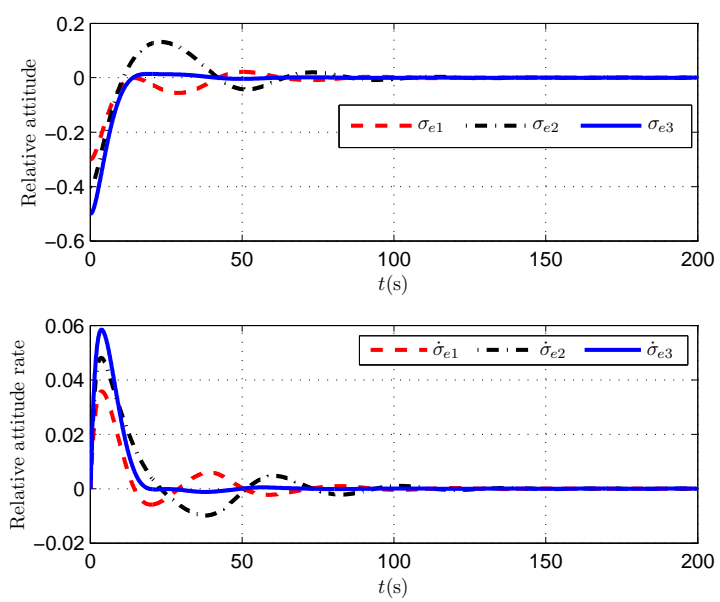

Fig. 4. Relative attitude and angular velocity under controller (14).
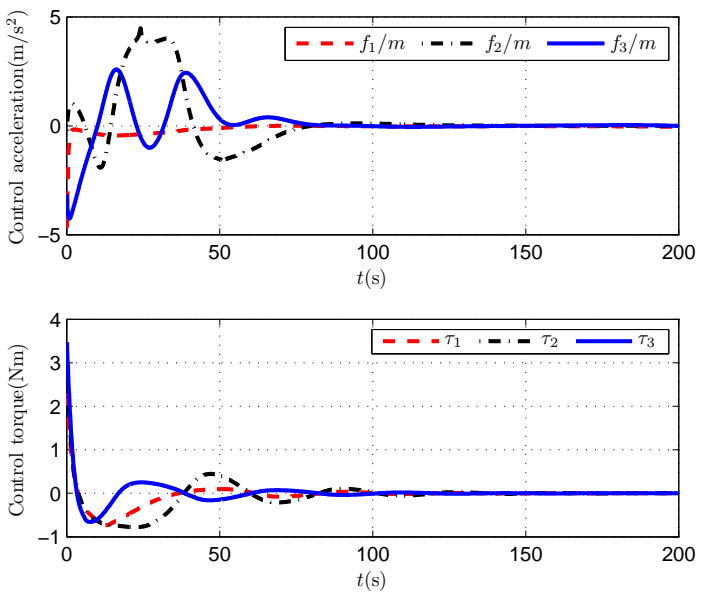

Fig. 5. Control accelerations and torques under controller (14).

\section{CONCLUSION}

A finite-time relative pose controller is developed for the cooperative rendezvous and docking systems subject to parametric uncertainties and unknown external disturbances. The robust control term is redesigned by employing a nonlinear exponential function to decrease the chattering phenomenon of the control inputs. In particular, the proposed controller can ensure that the relative pose and its velocity converge to zero in finite time and suppress the model uncertainties. Simulation results verify the effectiveness of the control systems. The proposed controller design method could be easily extended to more second-order nonlinear mechanical systems, such as robotic manipulators, aerial vehicles, underwater vehicles, and other space systems.

\section{ACKNOWLEDGMENT}

This work was supported by the National Natural Science Foundation of China (No. 61903025) and the China Scholarship Council (No. 201906465028).

\section{REFERENCES}

[1] R. Kristiansen and P. J. Nicklasson, "Spacecraft formation flying: a review and new results on state feedback control," Acta Astronaut., vol. 65, nos. 11-12, pp. 1537-1552, 2009.

[2] P. Singla, K. Subbarao, and J. L. Junkins, Adaptive output feedback control for spacecraft rendezvous and docking under measurement uncertainty," J. Guid. Control Dyn., vol. 29, no. 4, pp. 892-902, 2006.

[3] H. Yoon and B. N. Agrawal, "Novel expressions of equations of relative motion and control in Keplerian orbits," J. Guid. Control Dyn., vol. 32, no. 2, pp. 664-669, 2009.

[4] A. Weiss, M. Baldwin, R. S. Erwin, and I. Kolmanovsky, "Model predictive control for spacecraft rendezvous and docking: Strategies for handling constraints and case studies," IEEE Trans. Control Syst. Technol., vol. 23, no. 4, pp. 1638-1647, 2015.

[5] E. N. Hartley, G. Marco, and J. M. Maciejowski, "Terminal spacecraft rendezvous and capture with LASSO model predictive control," Int. J. Control, vol. 86, no. 11, pp. 2104-2113, 2013.

[6] G. Guglieri, F. Maroglio, P. Pellegrino, and L. Torre, "Design and development of guidance navigation and control algorithms for spacecraft rendezvous and docking experimentation," Acta Astronaut., vol. 94, no. 1, pp. 395-408, 2014.

[7] M. Navabi and M. R. Akhloumadi, "Nonlinear optimal control of relative rotational and translational motion of spacecraft rendezvous," J. Aerosp. Eng., vol. 30, no. 5, pp. 04017038, 2017.

[8] A. Imani and Beigzadeh, B. "Robust control of spacecraft rendezvous on elliptical orbits: optimal sliding mode and backstepping sliding mode approaches," Pro. Inst. Mechan. Eng., Part G: J. Aerosp. Eng., vol. 230, no. 10, pp. 1975-1989, 2016.

[9] E. Capello, P. Elisabetta, D. Fabrizio, G. Giorgio, and T. Roberto, "Sliding-mode control strategies for rendezvous and docking maneuvers," J. Guid. Control Dyn., vol. 40, no. 6, pp. 1481-1487, 2017.

[10] F. Zhang and G. Duan, "Robust adaptive integrated translation and rotation finite-time control of a rigid spacecraft with actuator misalignment and unknown mass property," Int. J. Syst. Sci., vol. 45, no. 5, pp. 10071034, 2014.

[11] D. Lee, A. K. Sanyal, E. A. Butcher, and D. J. Scheeres, "Finite-time control for spacecraft body-fixed hovering over an asteroid," IEEE Trans. Aerosp. Electron. Syst., vol. 51, no. 1, pp. 506-520, 2015.

[12] L. Sun, "Passivity-based adaptive finite-time trajectory tracking control for spacecraft proximity operations," J. Spacecraft Rockets, vol. 53, no. 1, pp. 46-56, 2016.

[13] C. Pukdeboon, "Extended state observer-based third-order sliding mode finite-time attitude tracking controller for rigid spacecraft," Sci. ChinaInfor. Sci., vol. 62, no. 1, pp. 012206, 2019.

[14] F. Bayat, "Model predictive sliding control for finite-time three-axis spacecraft attitude tracking," IEEE Trans. Ind. Electron., vol. 66, no. 10, pp. 7986-7996, 2019.

[15] G. Di Mauro, D. Spiller, S. F. R. Carna, and R. Bevilacqua, "Minimumfuel control strategy for spacecraft formation reconfiguration via finitetime maneuvers", J. Guid. Control Dyn., vol. 42, no. 4, pp. 752-768, 2019.

[16] J. Zhang, J. D. Biggs, D. Ye, and Z. Sun, "Finite-time attitude setpoint tracking for thrust-vectoring spacecraft rendezvous," Aerosp. Sci. Technol., vol. 96, pp. 105588, 2020.

[17] Y. Wang and H. Ji, "Integrated relative position and attitude control for spacecraft rendezvous with ISS and finite-time convergence," Aerosp. Sci. Technol., vol. 85, pp. 234-245, 2020.

[18] Q. Zhao and G. Duan, "Finite-Time concurrent learning adaptive control for spacecraft with inertia parameter identification," J. Guid. Control Dyn., vol. 43, no. 3, pp. 574-584, 2020.

[19] H. Schaub and J. L. Junkins, Analytical Mechanics of Space Systems, Reston: AIAA, 2003.

[20] A. H. J. De Ruiter and C. J. Damaren, "Effect of attitude parameterization on the perfermance of passivity-based adaptive attitude control," AIAA Guid., Nav. Control Conf. Exihib., Montreal, Canada, 2001, AIAA-2001-4154.

[21] R. Rascon, O. Penaloza-Mejia, J. Castro, "Improving first order sliding mode control on second order mechanical systems," European J. Control, vol. 29, pp. 74-80, May 2016.

[22] Q. Chen, S. Xie, M. Sun, and X. He, "Adaptive nonsingular fixedtime attitude stabilization of uncertain spacecraft," IEEE Trans. Aerosp. Electron. Syst., vol. 54, no. 6, pp.2937-2950, Dec. 2018. 